\title{
Size and emission wavelength control of InAs/InP quantum wires
}

\author{
David Fuster, ${ }^{2)}$ Luisa González, Yolanda González, and María Ujué González \\ Instituto de Microelectrónica de Madrid Centro Nacional de Microelectrónica-Consejo Superior \\ de Investigaciones Cientificas (CNM-CSIC), Isaac Newton 8, 28760 Tres Cantos, Madrid, Spain \\ Juan Martínez-Pastor \\ Instituto de Ciencia de los Materiales, Universidad de Valencia, P.O. Box 22085, 46071 Valencia, Spain
}

(Received 17 February 2005; accepted 13 June 2005; published online 3 August 2005)

\begin{abstract}
For a certain heteroepitaxial system, the optical properties of self-assembled nanostructures basically depend on their size. In this work, we have studied different ways to modify the height of InAs/InP quantum wires (QWrs) in order to change the photoluminescence emission wavelength. One procedure consists of changing the QWr size by varying the amount of InAs deposited. The other two methods explored rely on the control of As/P exchange process, in one case during growth of InAs on InP for QWr formation and in the other case during growth of InP on InAs for QWr capping. The combination of the three approaches provides a fine tuning of QWr emission wavelength between 1.2 and $1.9 \mu \mathrm{m}$ at room temperature. (C) 2005 American Institute of Physics.

[DOI: $10.1063 / 1.1996307]$
\end{abstract}

\section{INTRODUCTION}

InAs/InP quantum wires (QWrs) or quantum dots (QDs) are promising nanostructures for optoelectronic devices operating in the $1.30-1.55-\mu \mathrm{m}$ wavelength range employed in optical fiber telecommunication systems. ${ }^{1-3}$ However, up to now these nanostructures have not been so extensively exploited as InAs/GaAs QD. This is partly due to the intrinsic peculiarities associated with the interface between III$\mathrm{V}_{A} / \mathrm{III}-\mathrm{V}_{B}$ compounds: on one hand, an asymmetric stress appears at the InAs/InP interface, ${ }^{4,5}$ and on the other hand, complicated $\mathrm{V}_{A} / \mathrm{V}_{B}$ exchange processes take place during epitaxial growth. ${ }^{6,7}$ Both intrinsic properties have a strong influence on the nanostructures formation. So, the stress distribution is responsible for the final shape of the nanostructures, resulting either QWr [for InAs grown on $\operatorname{InP}(001)$ by molecular-beam epitaxy (MBE) $]^{4,5}$ or QD [for InAs grown on $\operatorname{InP}(311) \mathrm{B}] .{ }^{8}$ With regard to the As/P exchange processes, they are determinant for the final size of the nanostructures and hence for their optical properties since they basically depend on the size for a given heteroepitaxial system. In this way, the InAs/InP system possesses a huge flexibility, and taking advantage of it, could provide a large benefit in optoelectronic devices design. For example, the exchange processes are temperature dependent ${ }^{6,7}$ and they are also influenced by the strain distribution at the growth front. ${ }^{9}$ Thus, if appropriately controlled, they can be used as a design tool for the emission wavelength. Our aim in this work is to study different ways of controlling the size of MBE-grown InAs/InP(001) QWrs, establishing procedures to engineer the emission wavelength of these nanostructures.

In InAs/InP(001) QWrs, the height is the critical dimension for confinement (QWrs are typically $2 \mathrm{~nm}$ high, $12 \mathrm{~nm}$ wide, and about $1 \mu \mathrm{m}$ long) and consequently the emission wavelength will be strongly dependent on this geometrical parameter. The dependence of photoluminescence (PL) emis-

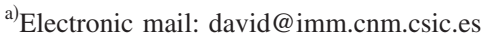

sion energy of InAs quantum wires on the QWr height has been studied in previous works. ${ }^{10,11}$ Within this perspective, our studies have been centered in the control of the QWr height by different procedures. On one hand, we have obtained QWrs of increasing height by deposition of different amounts of InAs above the critical thickness $\left(\theta_{c}\right)$ for $\mathrm{QWr}$ formation. ${ }^{3}$ On the other hand, we have exploited the As/P exchange processes to actuate on the QWr height. In this approach, we have considered that As/P exchange can take place in two different situations: As/P exchange when the InP surface is exposed to arsenic flux during InAs growth for QWr formation ${ }^{7}$ and $\mathrm{P} / \mathrm{As}$ exchange when the already formed InAs QWrs are exposed to phosphorus flux during InP cap layer growth. ${ }^{6}$

The PL results obtained in this work evidence the wide emission wavelength tuning range achieved by exploiting these different methods of InAs QWr size control. Moreover, this study demonstrates the interest of a deep understanding of the growth mechanisms in order to extract the maximum profit of nanostructures in device fabrication.

\section{EXPERIMENT}

All the samples studied in this work consist of InAs QWrs grown by $\mathrm{MBE}$ on $\mathrm{InP}(001)$ substrates. A 200-nm-thick InP buffer layer was grown before InAs deposition, and a 20-nm-thick InP cap layer was grown after InAs QWr formation. Several samples where the QWr were left uncovered were also grown for atomic force microscopy (AFM) characterization. Some growth parameters were common to all samples: beam equivalent pressure (BEP) $\mathrm{As}_{4}$ $=4.0 \times 10^{-6} \mathrm{mbar}$, BEP $\mathrm{P}_{2}=3.5 \times 10^{-6} \mathrm{mbar}$, and InP cap layer growth rate $r_{g}(\mathrm{InP})=1$ monolayer(ML)/s. Other growth parameters, such as $r_{g}$ (InAs), InAs coverage $\theta(\mathrm{InAs})$, substrate temperature for InAs growth $T_{\text {InAs }}$, or substrate temperature for InP cap layer growth $T_{\text {cap }}$, were changed as a function of the samples' design. Three series of samples, 
TABLE I. Growth parameters of the samples used in the photoluminescence studies: amount of InAs deposited $(\theta)$ expressed in monolayers (ML), InAs growth rate $\left(r_{g}\right)$ in $\mathrm{ML} / \mathrm{s}$, substrate temperature during InAs growth $\left(T_{\text {InAs }}\right)$, and substrate temperature during InP cap layer growth $\left(T_{\text {cap }}\right)$. The asterisk in $T_{\text {InAs }}$ values indicates that, after InAs deposition at that temperature, the samples were annealed at $515^{\circ} \mathrm{C}$ during $1 \mathrm{~min}$ of quantum wire $(\mathrm{QWr})$ formation

\begin{tabular}{ccccc}
\hline \hline Sample & $\begin{array}{c}\theta(\text { InAs }) \\
(\mathrm{ML})\end{array}$ & $\begin{array}{c}r_{g}(\mathrm{InAs}) \\
(\mathrm{ML} / \mathrm{s})\end{array}$ & $\begin{array}{c}T_{\text {InAs }} \\
\left({ }^{\circ} \mathrm{C}\right)\end{array}$ & $\begin{array}{c}T_{\text {cat }} \\
\left({ }^{\circ} \mathrm{C}\right)\end{array}$ \\
\hline A1 & 2.5 & 0.5 & $380^{*}$ & 380 \\
$\mathrm{~A} 2$ & 3.3 & 0.5 & $380^{*}$ & 380 \\
$\mathrm{~A} 3$ & 4.3 & 0.5 & $380^{*}$ & 380 \\
$\mathrm{~A} 4$ & 5.3 & 0.5 & $380^{*}$ & 380 \\
$\mathrm{~B} 1$ & 1.8 & 0.5 & 515 & 380 \\
$\mathrm{~B} 2 / \mathrm{C} 3$ & 1.6 & 0.1 & 515 & 380 \\
$\mathrm{C} 1$ & 1.6 & 0.1 & 515 & 515 \\
$\mathrm{C} 2$ & 1.6 & 0.1 & 515 & 470 \\
\hline \hline
\end{tabular}

designed for controlling the height and hence the emission wavelength of the QWr, have been considered.

Series A consists of four samples with different $\theta(\mathrm{InAs})=2.5,3.3,4.3$, and 5.3 ML (samples A1, A2, A3, and A4, respectively), while keeping constant $T_{\mathrm{InAs}}=380^{\circ} \mathrm{C}$, $r_{g}(\operatorname{InAs})=0.5 \mathrm{ML} / \mathrm{s}$, and $T_{\text {cap }}=380^{\circ} \mathrm{C}$. At these growth conditions, QWr formation was achieved by applying $1 \mathrm{~min}$ of thermal annealing at $515^{\circ} \mathrm{C}$ after InAs deposition.

Series B and C were designed for exploitation of As/P exchange processes. In this case $T_{\text {InAs }}$ was kept high enough $\left(T_{\text {InAs }}=515^{\circ} \mathrm{C}\right)$ to allow the formation of QWr during InAs deposition, so in these samples we could determine the InAs critical thickness for QWr formation $\left(\theta_{c} \sim 1.7 \mathrm{ML}\right)$ as detected by the two-dimensional (2D)-to-three-dimensional (3D) transition of the reflection high-energy electrondiffraction (RHEED) pattern.

In particular, series B consists of two samples where the variable parameter was the InAs growth rate: $r_{g}(\mathrm{InAs})=0.5$ and $0.1 \mathrm{ML} / \mathrm{s}$ (B1 and B2, respectively). The other growth parameters were kept constant $\left[\theta(\operatorname{InAs})=\theta_{c}, T_{\mathrm{InAs}}=515^{\circ} \mathrm{C}\right.$, and $\left.T_{\text {cap }}=380^{\circ} \mathrm{C}\right]$.

Series $\mathrm{C}$ consists of three samples where the changing parameter was the substrate temperature during InP cap layer growth: $T_{\text {cap }}=515,470$, and $380{ }^{\circ} \mathrm{C}(\mathrm{C} 1, \mathrm{C} 2$, and $\mathrm{C} 3$, respectively). The values for the other growth parameters, common to all the samples of the series were: $r_{g}(\mathrm{InAs})=0.1 \mathrm{ML} / \mathrm{s}$, $\theta(\operatorname{InAs})=\theta_{c}$, and $T_{\mathrm{InAs}}=515^{\circ} \mathrm{C}$.

The growth parameters used in the three series of samples described in this work are listed in Table I.

Contact mode AFM characterization has been performed in uncapped samples, as has been indicated. The emission wavelength of the InAs QWr has been assessed by PL measurements: the PL of the samples was excited by the 514.5$\mathrm{nm} \mathrm{Ar}^{+}$laser line, dispersed by a $0.22-\mathrm{m}$ focal length monochromator, and synchronously detected with a cooled Ge detector and an extended InGaAs photodiode.

\section{RESULTS AND DISCUSSION}

\section{A. QWr size control by the amount of InAs deposited}

Figure 1 shows the evolution of the QWr size with the amount of InAs deposited, studied by AFM characterization

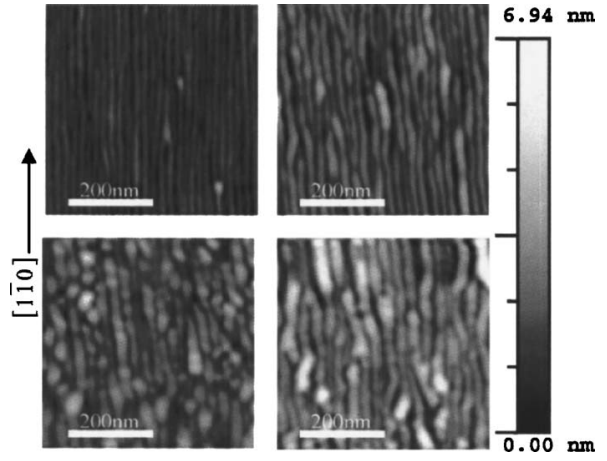

FIG. 1. $500 \times 500-\mathrm{nm}^{2} \mathrm{AFM}$ images of the four uncapped InAs quantum wire (QWr) samples with different InAs deposited thicknesses: $\theta$ (InAs) =2.5 ML, 3.3 ML, 4.3 ML, and 5.3 ML (from upper left to bottom right). The four images have the same $Z$-scale bar.

in uncapped samples of series A. For InAs deposited thicknesses of $\theta(\operatorname{InAs})=2.5,3.3,4.3$, and 5.3 ML, the QWr arrays show average periods of $18,23,29$, and $31 \mathrm{~nm}$, and average heights of 1.2, 1.8, 2.1, and $2.5 \mathrm{~nm}$, respectively. As can be seen in Fig. 1, the QWrs are oriented along [1 $1 \overline{1} 0]$ in all samples, and their overall size, width, and height increase with the nominal amount of InAs. Moreover, the InAs QWrs become more and more irregular with increasing InAs coverage. In this case, we can observe an increase of the density of 3D islands coexisting with the QWr. ${ }^{12}$ The formation of these QD-like nanostructures is probably related to local thickness fluctuations along the QWr: in some areas the local thickness becomes large enough to reach the critical thickness for elastic relaxation along the [1피 direction. In this situation, while the QWrs relax stress along [110] remaining fully strained along $[1 \overline{1} 0],{ }^{4,5}$ the QD-like nanostructures relax stress along both [110] and [1 $\overline{1} 0]$ directions. This hypothesis will be studied more carefully by means of in situ accumulated stress measurements in future experiments.

Figure 2 shows the PL spectra measured at $12 \mathrm{~K}$ of similar samples as those shown in Fig. 1, but covered in this case with a 20-nm-thick InP cap layer. We observe a redshift of the PL band as the InAs deposited thickness increases, changing the PL peak maximum from 1.40 to $1.75 \mu \mathrm{m}$. This change in the emission wavelength can be explained by the enlargement of the nanostructures' average height due to the bigger amount of InAs involved in the QWr formation (Fig. 1). At the same time, the measured values of the PL full width at half maximum (FWHM) clearly mark a frontier between the samples with $\theta(\operatorname{InAs})=3.3 \mathrm{ML}$ and $\theta(\operatorname{InAs})$ $=4.3 \mathrm{ML}$. For InAs coverage $\theta(\operatorname{InAs}) \leqslant 3.3 \mathrm{ML}$, we observe in the PL spectra narrow Gaussian lines $(25-35 \mathrm{meV})$ related to $1 \mathrm{ML}$ QWr height fluctuations, typical of this kind of samples. ${ }^{1,10}$ Conversely, for $\theta(\operatorname{InAs}) \geqslant 4.3 \mathrm{ML}$, different contributions to PL due to $1 \mathrm{ML}$ fluctuations in the wire height are no longer resolved, thus giving rise to a very broad emission band. These results can be perfectly correlated with the AFM images in Fig. 1, in the sense that the observed broadening of the PL spectra for $\theta(\operatorname{InAs}) \geqslant 4.3 \mathrm{ML}$ is due to the more important disorder and inhomogeneity in the QWr morphology by increasing the InAs amount, as was discussed above. While the increase of the QWr height was the origin 


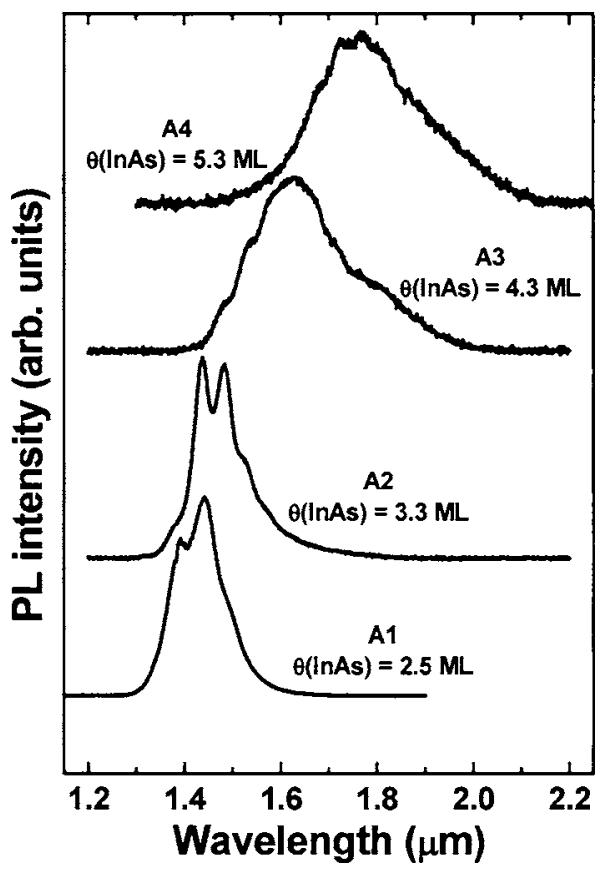

FIG. 2. 12-K photoluminescence (PL) spectra of InAs QWrs formed after deposition of 2.5, 3.3, 4.3, and 5.3 ML of InAs.

of the PL band redshift, the size fluctuations and the appearance of 3D islands would cause the PL band broadening. It should be noted that, in spite of the broad emission band observed in these samples, these structures could be included in the active region of long-wavelength laser devices, for which tuning of some special wavelength is needed. ${ }^{3}$

\section{B. QWr size control by the InAs deposition rate}

Figure 3 shows the PL spectra of the two samples included in series B, where we have changed the InAs growth rate. We observe that the increase of the InAs deposition rate, keeping the rest of the growth parameters identical, results in a net blueshift of the measured PL band. The comparable FWHM of the PL bands in all cases point out that the homo-

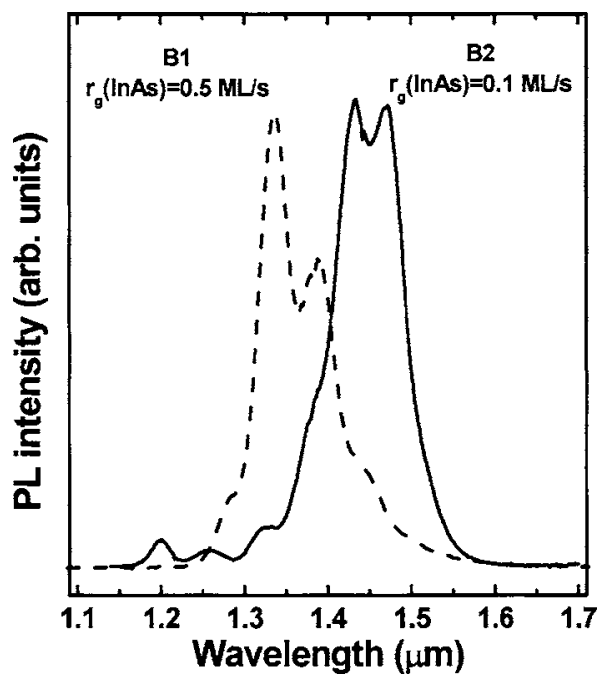

FIG. 3. 12 K-PL spectra of InAs QWrs grown at different InAs growth rates $\left[r_{g}(\mathrm{InAs})=0.1\right.$ and $\left.0.5 \mathrm{ML} / \mathrm{s}\right]$.

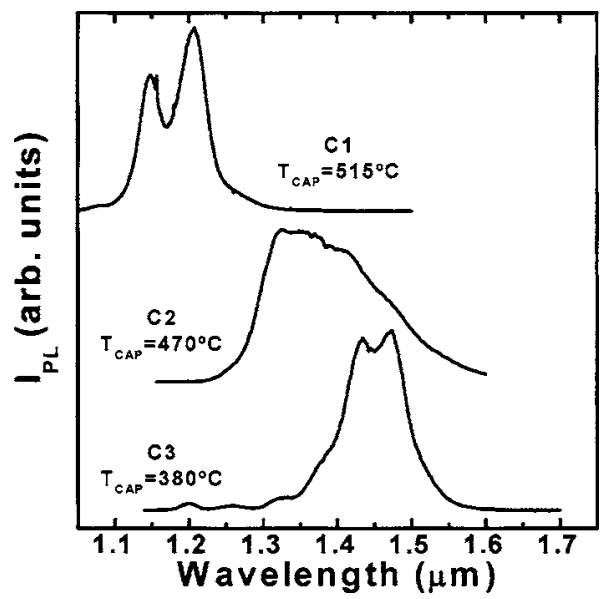

FIG. 4. 12-K PL spectra of InAs QWr samples where the InP cap layer has been grown at different substrate temperatures $\left(T_{\text {cap }}=380,470\right.$, and $\left.515^{\circ} \mathrm{C}\right)$.

geneity and uniformity of the QWr size distribution is very similar in both samples. The observed changes in the PL energy indicate their different average QWr height. Considering that two consecutive Gaussian components are associated to a height variation of $1 \mathrm{ML}$, the QWrs in the sample grown at $r_{g}(\mathrm{InAs})=0.1 \mathrm{ML} / \mathrm{s}$ (sample B2) are 2-3 ML higher than those in the sample grown at $r_{g}(\operatorname{InAs})$ $=0.5 \mathrm{ML} / \mathrm{s}$ (sample B1). We propose that the exchange between As and P atoms, at the InP surface exposed to arsenic flux during InAs growth, is responsible for this behavior. During InAs deposition previous to QWr formation, both arsenic and indium atoms are delivered at the InP surface. The lower the indium deposition rate is, the longer InP the surface is exposed to arsenic before one InAs ML has been completely deposited. In this period, the As/P exchange process operates indeed as an indium source at the expense of the InP buffer layer, resulting in an effective InAs growth rate that is higher than the deposition rate imposed by the temperature of the indium cell. This extra amount of incorporated InAs leads to the formation of higher QWr with emission at longer wavelengths, especially by using slow growth rates (as observed in Fig. 3 for the sample grown at $0.1 \mathrm{ML} / \mathrm{s}$ ). Obviously, the QWr emission wavelength could be more precisely tuned by changing progressively the InAs growth rate.

Moreover, these results show that the actual InAs critical thickness for the QWr formation is not only determined by the amount of InAs nominally deposited. It is necessary to consider also the additional quantity of InAs incorporated at the InP surface, as a result of the complicated dynamics of the molecules at the growth front. In fact, the InAs QWr can be formed by As/P exchange after a long-time exposure of an InP surface to an arsenic flux even without indium deposition. ${ }^{9,13}$

\section{Size control by changing the InP cap layer growth temperature}

Figure 4 shows the PL measurements in samples of series $\mathrm{C}$, where we used different substrate temperatures $\left(T_{\text {cap }}\right)$ during InP cap layer growth. We see that the measured PL spectrum shifts to shorter wavelengths as $T_{\text {cap }}$ increases from 
380 to $515{ }^{\circ} \mathrm{C}$. This energy shift of the whole PL band can be explained considering that $T_{\text {cap }}$ modifies the P/As exchange ratio at the interface between the InAs QWr and the InP cap layer, thus affecting the final height of the InAs QWr. In this way, the higher the $T_{\text {cap }}$ the more important the P/As exchange ratio becomes. In other words, we will obtain smaller InAs QWr for higher $T_{\text {cap }}$.

In a previous work we studied in detail the P/As exchange process at the InP/InAs interface, demonstrating that the surface reconstruction of the InAs surface, which depends on the substrate temperature for a fixed arsenic pressure, determines the P/As exchange amount. ${ }^{6}$ When the InP cap layer is grown at $T_{\text {cap }}=380{ }^{\circ} \mathrm{C}$, the InAs surface shows an As-rich reconstruction $[\gamma(2 \times 4)$ corresponding to an arsenic surface coverage of $1 \mathrm{ML}],{ }^{6,14}$ both in the presence of arsenic flux and immediately after the arsenic flux is stopped. This indicates that arsenic desorption is negligible at this temperature. In this situation, phosphorus atoms do not easily attach to that arsenic-terminated surface, since no indium atoms are available for chemical bonding. So, at $380{ }^{\circ} \mathrm{C}$ (as well as at all low temperatures where surface presents this surface reconstruction) $\mathrm{P} / \mathrm{As}$ exchange process is negligible and consequently no change in $\mathrm{QWr}$ size should be expected during capping with InP.

By increasing the substrate temperature, arsenic desorption becomes more important and the InAs surface reconstruction evolves towards more In-rich configurations. So, at $T_{\text {cap }}=470{ }^{\circ} \mathrm{C}$ the InAs surface reconstruction with the As cell opened is still $\gamma(2 \times 4)$, but it quickly changes to $\alpha(2 \times 4)$ (corresponding to an arsenic surface coverage of $0.5 \mathrm{ML}$ ) when the As cell is closed. At $T_{\text {cap }}=515{ }^{\circ} \mathrm{C}$, the InAs surface reconstruction is $\alpha(2 \times 4)$ under As flux, which suddenly evolves to an In-rich $(4 \times 2)$ surface when the As cell is closed. Then, we expect an increase of phosphorus incorporated at the growth front at the expense of the As lost from the QWr, which leads to a reduction of their size. All these expected changes in the average QWr height are in agreement with the detected blueshift of the central PL energy with increasing $T_{\text {cap }}$ shown in Fig. 4. Since surface reconstructions, with their corresponding In and As surface converges, exhibit a rather steplike evolution with the substrate temperature, we would also expect discontinuous changes for the PL peak wavelength by varying $T_{\text {cap }}$, as is actually observed.

On the other hand, as explained above concerning the As/P exchange dependence on the InAs growth rate, the control of the growth rate of the InP layer used to cap the InAs QWr can also be used for further controlling the P/As exchange. ${ }^{15}$

\section{SUMMARY AND CONCLUSIONS}

We have reported three different ways to change the PL emission wavelength of InAs/InP QWr by modifying their height during the growth process. One of the reported methods consists of varying the amount of deposited InAs. The other two procedures explored rely on the control of As/P exchange processes during growth of InAs/InP for QWr formation and during $\mathrm{InP} / \mathrm{InAs}$ growth for capping the $\mathrm{QWr}$.

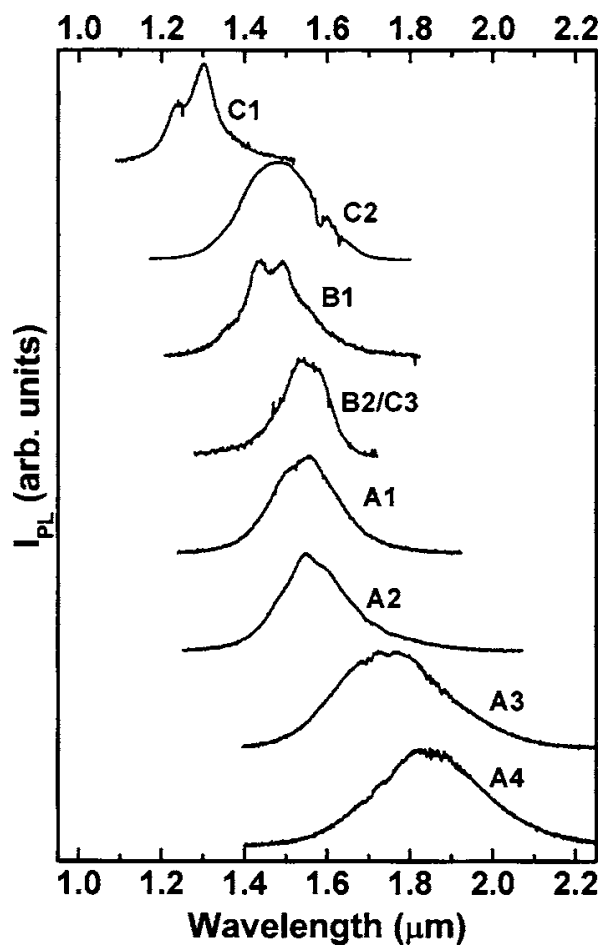

FIG. 5. Room-temperature PL spectra of the three series of InAs QWr samples studied in this work (see text and Table I for the description of the samples).

Taking advantage of As/P exchange at both interfaces, we have demonstrated that the QWr size can be changed by using different InAs growth rates and/or different substrate temperature during InP cap layer growth.

In summary, our experimental results show that it is possible to achieve a large redshift by increasing the InAs deposited thickness, a fine tuning of emission wavelength by changing the InAs growth rate, and a steplike variation of emission wavelength by changing the substrate temperature during InP cap layer growth. All at once, the combination of the three approaches allows to tune the QWr emission inside a large wavelength range of $1.2-1.9 \mu \mathrm{m}$ at room temperature (see Fig. 5).

The main drawback for emission extension towards longer wavelengths is the broadening of the emission peaks that were obtained for larger InAs coverage, as discussed in Sec. III A. However, it leads to the formation of highly nonuniform, wide and meandering $\mathrm{QWr}$ size distributions and also 3D islands (or QD-like structures), as was shown in the AFM images of Fig. 1. The coexistence of both kinds of nanostructure ensembles produces the observed PL band broadening (samples A3 and A4). In spite of such obtained broad PL bands, it is remarkable that the extension of the emission towards very long wavelengths in the InAs/InP system, at ranges far from those accessible by using quantum wells, is particularly useful for some kind of laser diodes. ${ }^{3}$

\section{ACKNOWLEDGMENTS}

This work was financed by Spanish MCyT under NANOSELF project (TIC2002-04096) and MAT2003-02655 
and by the SANDiE Network of excellence (Contract No. NMP4-CT-2004-500101 group TEP-0120).

${ }^{1}$ L. González, J. M. García, R. García, F. Briones, J. Martínez-Pastor, and C. Ballesteros, Appl. Phys. Lett. 76, 1104 (2000).

${ }^{2}$ D. Fuster, L. González, Y. González, J. Martínez-Pastor, T. Ben, A. Ponce, and S. I. Molina, Eur. Phys. J. B 40, 433 (2004).

${ }^{3}$ R. Schwertberger, D. Gold, J. P. Reithmaier, and A. Forchel, J. Cryst. Growth 251, 248 (2003).

${ }^{4}$ J. M. García, L. González, M. U. González, J. P. Silveira, Y. González, and F. Briones, J. Cryst. Growth 227, 975 (2001).

${ }^{5}$ M. U. González, L. González, J. M. García, Y. González, J. P. Silveira, and F. Briones, Microelectron. J. 35, 13 (2004).

${ }^{6}$ D. Fuster, M. U. González, L. González, Y. González, T. Ben, A. Ponce, S. I. Molina, and J. Martínez-Pastor, Appl. Phys. Lett. 85, 1424 (2004).
${ }^{7}$ M. U. González, J. M. García, L. González, J. P. Silveira, Y. González, J. D. Gómez, and F. Briones, Appl. Surf. Sci. 188, 188 (2002).

${ }^{8}$ C. Paranthoen et al., J. Cryst. Growth 251, 230 (2003).

${ }^{9}$ D. Fuster, M. U. González, L. González, Y. González, T. Ben, A. Ponce, and S. I. Molina, Appl. Phys. Lett. 84, 4723 (2004).

${ }^{10}$ B. Alén, J. Martínez-Pastor, A. García-Cristóbal, L. González, and J. M. García, Appl. Phys. Lett. 78, 4025 (2001).

${ }^{11}$ J. Maes et al., Phys. Rev. B 70, 155311 (2004).

${ }^{12}$ H. R. Gutiérrez, M. A. Cotta, and M. M. G. de Carvalho, Mater. Res. Soc. Symp. Proc. 707, N5.7.1 (2002).

${ }^{13}$ H. Yang, P. Ballet, and G. J. Salamo, J. Appl. Phys. 89, 7871 (2001).

${ }^{14}$ K. B. Ozanyan, P. J. Parbrook, M. Hopkinson, and C. R. Whitehouse, Thin Solid Films 364, 6 (2000).

${ }^{15}$ P. J. Poole, R. L. Williams, J. Lefebvre, and S. Moisa, J. Cryst. Growth 257, 89 (2003). 